\title{
reviscafuences
}

ISSN: 1575.7072 | 8.15SN: 2172.7775

Páginas: 254-267

Recibido: 2020-06-28

Revisado: 2020-07-09

Aceptado: 2021-04-16

Preprint: 2021-01-05

Publicación Final: 2021-15-05

www.revistascientificas.us.es/index.php/fuentes/index

DOI: https://doi.org/10.12795/revistafuentes.2021.12108

\section{Estudio sobre la inteligencia emocional en educación primaria}

\section{Study on emotional intelligence in primary school}

\author{
(iD) Ingrid Arely Godoy Rojas \\ Universidad de Sevilla (España) \\ (iD) Marita Sánchez Moreno \\ Universidad de Sevilla (España)
}

\begin{abstract}
Dada la relevancia de las competencias emocionales en el proceso de enseñanza-aprendizaje, se presenta este artículo cuyo propósito es comparar, en función del género, los niveles de inteligencia emocional del alumnado de 5 to. básico de las escuelas de Temuco (Chile). La investigación se enmarca en el paradigma positivista, nivel descriptivo, diseño no experimental y transeccional. Para lograr el objetivo se aplicó la escala Trait Meta Mood Scale-24 (TMMS-24) a una muestra de 450 estudiantes de 12 escuelas de Temuco. La mayoría de los estudiantes presenta niveles adecuados de inteligencia emocional en las tres dimensiones de la escala: atención, claridad y regulación emocional. Respecto de los niveles de inteligencia emocional de hombres y mujeres, no se encontraron diferencias estadísticamente significativas en las dimensiones de atención y regulación emocional; en cambio, en la dimensión de claridad emocional las mujeres se ubican en un nivel superior al de los hombres. En cuanto a la relación entre inteligencia emocional y género, existe relación estadísticamente significativa entre género y atención y claridad emocional, pero en la dimensión reparación no se observó tal relación. En conclusión, como no se hallaron resultados concluyentes sobre la relación entre los niveles de inteligencia emocional y el género, se sugiere realizar más investigación.
\end{abstract}

\section{Abstract}

Given the relevance of emotional competences in the teaching-learning process, the article is presented, to compare, in term of gender, the levels of emotional intelligence of students in the 5th grade of schools in Temuco (Chile). The design of the research is framed in the positivist paradigm, descriptive level, non-experimental and transactional. To achieve the objective, the Trait Meta Mood Scale-24 (TMMS-24) was applied to a sample of 450 students from 12 schools in Temuco. Most of the students present adequate levels of emotional intelligence in the three dimensions of the scale: attention, clarity, and emotional regulation. Regarding the levels of emotional intelligence of men and women, no statistically significant differences were found in the dimensions of emotional attention and regulation; on the other hand, in the dimension of emotional clarity, women are at a higher level than men. Regarding the relationship between emotional intelligence and gender, there are statistically significant differences in emotional attention and clarity, but no such relationship was observed in the emotional regulation. As no conclusive results were found on levels the relationship between emotional intelligence and gender, further research is suggested.

\section{Palabras clave}

Inteligencia emocional, educación, género, escuelas públicas, estudiante, mujer, hombre.

\section{Keywords}

Emotional intelligence, education, gender, public schools, student, woman, man. 


\section{Introducción}

El interés por realizar este estudio sobre la inteligencia emocional (IE) surgió de las observaciones de las interacciones al interior del aula entre profesores y estudiantes, sus repertorios sociales emocionales, las características del profesor, las diferencias emocionales entre mujeres y hombres, dejando a la vista una serie de problemas educativos complejos. La propuesta de Pulido y Herrera (2017) de incorporar el componente emocional en el proceso de enseñanza-aprendizaje para lograr mayor eficacia y calidad también motivó al desarrollo de este estudio.

La IE se define como una competencia para manejarse a sí mismo y sus relaciones con los demás, trabajar en equipo efectivo y asumir liderazgo, la cual tiene efectos positivos en términos de productividad y rendimiento académico y laboral (Goleman, 1995). Es la capacidad de reconocer nuestros propios sentimientos y los de los demás, motivarnos y manejar bien las emociones en nosotros mismos y en nuestras relaciones (Goleman, 1998). Según este autor, las investigaciones sobre la prueba de $\mathrm{Cl}$ han descubierto que este representa el $20 \%$ del éxito en la vida de una persona, lo cual sugiere que la IE puede estar asociada con el $80 \%$ restante. Por ello, el estudio de la IE es esencial en el ámbito educativo para lograr que los estudiantes se formen integralmente y desarrollen todas sus potencialidades (Goleman, 1997).

La IE ha sido ampliamente estudiada en estudiantes de diferentes niveles educativos de diferentes países empleando diferentes pruebas, como se describe más adelante. En Chile, los estudios de la IE son menos numerosos. Gómez et al. (2020) analizaron la asociación entre el género y la IE en adolescentes, GarcíaFernández et al. (2015), Díaz-Herrero et al. (2018) también estudiaron la IE en adolescentes, pero no incluyeron el género como variable de estudio. Sin embargo, hasta la fecha no se han analizado la relación de la IE y el género en niños de educación primaria de escuelas públicas de Temuco, Chile.

Se seleccionó el 5to. año básico, pues en este período los educandos se encuentran en una etapa de transición desde la niñez a la preadolescencia, gradualmente van abandonando el funcionamiento infantil y tienen una serie de cambios físicos y emocionales (Lillo, 2004). Además, Traveset (2016) plantea que, entre los 6 y los 12 años, comienza la vida interior en la que se diferencia el yo del mundo fantástico. En esta etapa se adquiere y desarrolla la lectura, la escritura, el cálculo matemático, entre otros, el niño va procesando y a la vez elaborando su propia valoración: si el proceso es exitoso, se generan sentimientos de autoestima y se potencian las propias capacidades; en caso contrario, se instalará un sentimiento de inferioridad.

\section{Objetivo e hipótesis}

Esta investigación tuvo como objetivo analizar y comparar las dimensiones de inteligencia emocional del alumnado de 5to. básico de las escuelas públicas de Temuco en función del género. Para lograr este propósito, se plantearon tres hipótesis de trabajo: 1) Los estudiantes de 5to. básico presentan niveles adecuados de inteligencia emocional en función de las dimensiones de la escala TMMS-24, 2) Las mujeres presentan mejores niveles de IE que los hombres en las tres dimensiones, 3) No existe relación entre los niveles de cada dimensión y el género.

\subsection{Variables}

En este estudio, el género es la variable independiente y los niveles de inteligencia emocional, la dependiente. El género se refiere a los conceptos sociales de las funciones, comportamientos, actividades y atributos que cada sociedad considera apropiados para los hombres y las mujeres (OMS, 2021). Para efectos de este artículo, se emplearán indistintamente los términos mujer y hombre o femenino y masculino. La variable niveles de IE se entiende operacionalmente como las dimensiones establecidas en la escala TMMS-24 de Mayer y Salovey $(1993,2007)$, adaptada al español por Fernández-Berrocal et al. (1998), cuyos valores están establecidos en función de los rangos de las dimensiones de atención, claridad y regulación.

\subsection{Modelos de inteligencia emocional}

En 1990, Salovey y Mayer (1990) emplearon por primera vez el término "inteligencia emocional" para referirse a la capacidad de percibir y expresar emociones, asimilar la emoción en el pensamiento, comprender y razonar con la emoción, y regular la emoción en uno mismo y en los demás (Mayer \& Salovey, 1993, 2007). Posteriormente, Fernández-Berrocal y Extremera (2005) la definieron como una habilidad centrada en el procesamiento de la información que conecta el razonamiento y las emociones, permitiendo una mejor adaptación a un ambiente en continuo cambio. 
Mayer y Salovey $(1997,2007)$ clasificaron los postulados de la IE en dos grandes modelos: modelos de habilidades y modelos mixtos. En el modelo de habilidades, llamado inteligencia emocional percibida (IEP) o autoinformada, la IE está formada por el conjunto de habilidades propias de la persona diferenciadas de la personalidad, rasgos cognitivos y de las potencialidades sociales. La IE corresponde a la habilidad de unir el pensamiento cognitivo y las emociones, orientando las emociones para facilitar un pensamiento y funcionamiento efectivos (Extremera \& Fernández Berrocal, 2005; Extrema et al., 2006).

Por otra parte, de acuerdo con los modelos mixtos, la IE está formada por diversas habilidades, destrezas, rasgos de personalidad, competencias y disposiciones afectivas. Entre este grupo, los más representativos son los modelos de Daniel Goleman y Reuven Bar-On (Fragoso-Luzuriaga, 2015). Ugarriza y Pajares (2005) sostienen que estos modelos son multifactoriales, ya que consideran variables cognitivas, medioambientales, afectivas y de personalidad. En este contexto, Goleman $(1995,1997,1998)$ indica que tenemos dos mentes: la racional y la emocional. La mente racional es la forma de comprensión desde la consciencia, reflexiva y capaz de analizar y meditar, y la emocional, impulsiva e incluso ilógica. Ambas representan la dicotomía clásica entre corazón y cabeza. Sin embargo, estas teorías de la IE tienen limitaciones importantes para su aplicación en ámbitos educativos, pues como lo señalan Belli et al. (2020), Palmer et al. (2005) y Pal y Baruah (2021), estudian las emociones solo desde una perspectiva cuantitativa, psicológica e individual, aunque las emociones también involucran aspectos socioculturales de carácter colectivo.

Así, en las últimas décadas ha aumentado el interés por estudiar biología y la anatomía de las emociones para comprender las conductas emocionales individuales (Ruiz, 2013). En esta línea, Maturana (2001) indicó que "Todo sistema racional se constituye en el operar con premisas aceptadas a priori desde cierta emoción" (p. 8) y postuló la interrelación entre pensamiento y emoción, dando prioridad al control de las emociones sobre la cognición. Respecto de las emociones en educación, planteó que debemos vivir nuestro educar, de modo que el niño aprenda a aceptar y a respetarse a sí mismo, porque así aprenderá a aceptar y respetar a los otros.

De acuerdo con Mayer y Salovey $(1997,2007)$, las dimensiones de la IE son: atención, claridad y reparación o regulación emocional:

La atención se refiere a la capacidad de percibir los sentimientos propios en el mismo momento que se dan, es decir, el registro de los estímulos emocionales en sí mismo. Está compuesta por dos capacidades, percepción emocional personal, que busca que la persona dirija y mantenga la atención sobre uno mismo y, percepción emocional interpersonal que consiste en disponer de una óptima conciencia de las emociones de los demás, es decir, poder identificar las señales corporales de los otros.

Por su parte, la claridad emocional es la habilidad para identificar y comprender los propios estados emocionales reconociendo la causa que los genera. Integra la emoción, la razón y una competencia social que es la empatía, lo cual permite contar con inteligencia y, por ende, tomar decisiones acertadas, mientras se posiciona emocionalmente en el lugar de la otra persona y es consciente de sus sentimientos y las implicaciones que pudiera tener en su vida.

Por último, la dimensión de reparación emocional se refiere a la capacidad que tiene la persona de impedir los efectos negativos de una emoción y utilizar sus aspectos positivos con la finalidad de actuar sin perjudicarse. Va dirigida hacia uno mismo y hacia los demás. Por ello, encontramos la regulación emocional personal, que es la capacidad para no generar respuestas emocionalmente descontroladas, y la resolución de conflictos interpersonales, habilidad para relacionarse con los demás, saber escuchar y saber responder en el momento oportuno, emplear la crítica constructiva, tener honestidad, llegar a acuerdos y saber defender las ideas propias respetando a los demás.

\subsection{Inteligencia emocional y género}

Los resultados de los estudios que han analizado comparativamente la IE y el género no indican una clara tendencia en cuanto a la asociación de ambas variables. Esto se debe, probablemente, a que se usan distintos instrumentos, lo cual dificulta identificar regularidades. Algunos estudios han encontrado que hay diferencias de género, entre las puntuaciones de la IE de los niños y las niñas; los niños obtuvieron puntajes superiores que las niñas (Ferrándiz et al., 2012; Khan \& Bhat, 2013; Pal \& Baruah, 2021), lo que también se reflejó en adultos jóvenes (Bindu \& Thomas, 2006; Shahzad \& Bagum, 2012).

Otros, en cambio, han hallado diferencias de género en cuanto al nivel de IE en niños, adolescentes y adultos. Los resultados indican que las mujeres obtienen mejores puntuaciones que los hombres (Extremera et al., 2006; Santesso et al., 2006; Joseph \& Newman, 2010; Fernández-Berrocal et al., 2012; Salguero et al., 2010, 2012; Billings et al., 2014; Cabello et al., 2016; Herrera et al., 2017). Brody y Hall (2000), y MartínezMarín y Martínez (2016) encontraron que las mujeres tienen una mayor comprensión emocional, ya que pueden expresar emociones tanto positivas como negativas con mayor facilidad, tienen mejores competencias interpersonales, son más competentes socialmente y pueden percibir y comprender las emociones mejor que 
los hombres. Por su parte, Jausovec y Jausovec (2005) encontraron diferencias de género en la ejecución de tareas emocionales, siendo las mujeres más competentes en su desempeño. Merchán (2017) también observó que, entre 11 y 12 años, se aprecia un ligero aumento de las puntuaciones a favor del género femenino. Similarmente, Garay (2014) encontró que los resultados globales son mejores para las mujeres que para los hombres, pero en la relación de claridad emocional obtuvieron puntuaciones más bajas y en reparación emocional las puntuaciones fueron semejantes para ambos géneros.

Por otro lado, los resultados de otras investigaciones que también compraran IE y género en niños, adolescentes y adultos no han hallado diferencias estadísticamente significativas, lo cual sugiere que no hay relación entre el género y la IE (McKinley et al., 2014; Salavera et al., 2017; Fischer et al., 2018; Mokhlesi \& Patil, 2018). Similarmente, Katyal y Awasthi (2005) y Williams et al. (2009) encontraron que las niñas obtuvieron puntajes ligeramente superiores que los niños, pero las diferencias no fueron estadísticamente significativas. Aunque algunos estudios han encontrado que las mujeres presentan mayores niveles de IE que los hombres (Gartzia et al., 2012; Pulido \& Herrera, 2017; Sánchez et al., 2008; Valadez et al., 2013), su autopercepción tiende a ser más baja que la de los hombres (Sánchez et al., 2008, p. 465). Considerando las habilidades verbales, las niñas son más diestras en la expresión de sus sentimientos y palabras debido a que disponen de recursos verbales que les permiten reemplazar las peleas físicas por el diálogo, el uso de términos emocionales para confrontar, argumentar y evitar los conflictos. En este sentido, es importante considerar las diferencias que tradicionalmente se atribuyen a niños y niñas y cómo esas diferencias influyen en la visión de mundo que cada uno tiene. Sánchez et al. (2008) plantean que, al buscar el origen de estas diferencias, hay que ir a la infancia y analizar las formas de crianza.

\section{Metodología}

La investigación se basa en el enfoque metodológico cuantitativo, es de alcance descriptivo correlacional y de diseño transeccional, no experimental (Hernández et al., 2014). El propósito de los estudios de este alcance "es describir variables y analizar su incidencia e interrelación en un momento dado. Es como "tomar una fotografía de algo que sucede" (Hernández et al., 2014, p. 154). Se optó por este nivel de investigación, ya que se pretende describir y comparar cuantitativamente los niveles de las dimensiones de atención, claridad y regulación de IE que poseen los estudiantes de 5to. básico de Temuco, y a la vez se intenta determinar la relación entre dichos resultados y la variable género.

\subsection{Población y Muestra}

La población estuvo formada por 985 estudiantes de 5to. año básico de 19 escuelas urbanas de Temuco, Chile. La muestra se obtuvo mediante un muestreo no probabilístico de tipo intencional de 12 establecimientos, de las cuales se seleccionaron niños y niñas de 5to. año básico, cuyas edades oscilaban entre 10 y 12 años. La muestra inicial formada por 458 estudiantes, luego de aplicar la tasa de no respuesta y eliminar ocho cuestionarios por inconsistencia por omisión de dos o más respuestas de la escala, quedó en 450 estudiantes: 224 mujeres, correspondiente al $49,8 \%$, y 226 hombres, 50,2\%. Para un mayor conocimiento de la muestra, en la Figura 1 se presenta su clasificación considerando los criterios grupo socioeconómico (GSE) de las familias y clasificación categoría desempeño (CCD). Al respecto, se valora el planteamiento de Alonso et al. (2018), quienes plantean que la situación económica familiar determina en parte la inteligencia emocional.

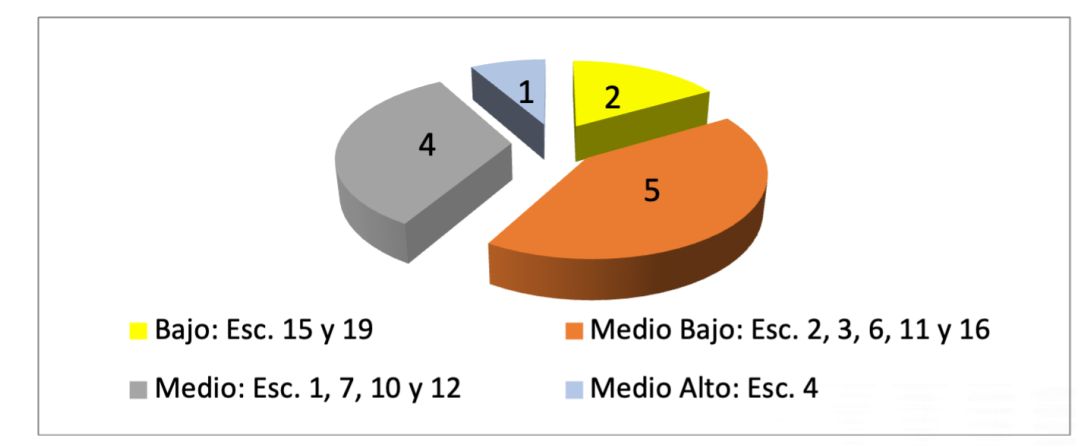

Figura 1. Clasificación socioeconómica GSE de las escuelas de Temuco 
Por otra parte, la CCD corresponde al resultado de una evaluación integral que considera los resultados de la prueba SIMCE y los Indicadores de Desarrollo Personal y Social (IDPS) de las escuelas- comprende cuatro desempeños: Alto, medio, medio-bajo e insuficiente (Agencia Calidad de la Educación, 2017). En el caso de nuestro estudio, de los 12 establecimientos participantes el $50 \%$ se clasifican en la categoría de desempeño medio-bajo y $50 \%$ en nivel medio, ninguna de las escuelas de esta muestra se ubica en los niveles insuficiente y alto (Figura 1).

\subsection{Instrumento}

El instrumento utilizado fue la Escala EIP denominada Trait Meta Mood Scale-24 (TMMS-24), adaptada al castellano (Fernández-Berrocal et al., 2004; Extremera \& Fernández-Berrocal, 2005) a partir del modelo de Salovey et al. (995). Dicha escala (Figura 2) está compuesta por 24 ítems, valorados por una escala tipo Likert según la cual la puntuación 1 es "nada de acuerdo" y 5, "totalmente de acuerdo". Como se observa en la Tabla 1 , se evalúan las tres dimensiones incluidas la escala original: atención, claridad y regulación emocional.

\section{Tabla 1}

Nivel de calificación del Instrumento de la Inteligencia Emocional

\begin{tabular}{|l|l|l|} 
Dimensión & $\begin{array}{l}\text { Mujeres } \\
\text { Debe mejorar su atención: presta } \\
\text { poca atención } \leq 24 \\
\text { Adecuada atención } 25 \text { a } 35 \\
\text { Debe mejorar su atención: presta emocional } \\
\text { demasiada atención } \geq 36\end{array}$ & $\begin{array}{l}\text { Hombres } \\
\text { Debe mejorar su atención: presta poca } \\
\text { atención } \leq 21 \\
\text { Adecuada atención 22 a } 32 \\
\text { Debe mejorar su atención: presta } \\
\text { demasiada atención } \geq 33\end{array}$ \\
Claridad o comprensión emocional & $\begin{array}{l}\text { Debe mejorar su comprensión } \leq 23 \\
\text { Adecuada comprensión 24 a 34 } \\
\text { Excelente comprensión } \geq 35\end{array}$ & $\begin{array}{l}\text { Debe mejorar su comprensión } \leq 25 \\
\text { Adecuada comprensión } 26 \text { a } 35 \\
\text { Excelente comprensión } \geq 36\end{array}$ \\
Reparación o regulación emocional & $\begin{array}{l}\text { Debe mejorar su regulación } \leq 23 \\
\text { Adecuada regulación } 24 \text { a } 34 \\
\text { Excelente regulación } \geq 35\end{array}$ & $\begin{array}{l}\text { Debe mejorar su regulación } \leq 23 \\
\text { Adecuada regulación } 24 \text { a } 35 \\
\text { Excelente regulación } \geq 36\end{array}$ \\
\hline
\end{tabular}

\section{Estudio psicométrico del instrumento}

Como se muestra en la Tabla 2, la consistencia interna del total de preguntas calculada mediante el Alfa de Cronbach obtuvo un $87 \%$. Respecto de las medidas globales obtenidas, como se muestra en la Tabla 3 , fueron superiores a $70 \%$. Por su parte, la medida de la dimensión atención emocional fue $72 \%$, claridad emocional, 77 $\%$ y reparación emocional, $78 \%$. Estos resultados confirman que los ítems son homogéneos y que la escala mide de forma consistente el objeto de estudio, las dimensiones de la IE en estudiantes de 10 a 12 años.

Tabla 2

Estadística de fiabilidad total del TMMS-24

\begin{tabular}{|l|l|l|}
\hline Alfa de Cronbach & $\begin{array}{l}\text { Alfa de Cronbach basada en elementos estandarizados } \\
0,869\end{array}$ & $\begin{array}{l}\mathrm{N} .{ }^{\circ} \text { de elementos } \\
24\end{array}$ \\
\hline
\end{tabular}

\section{Tabla 3}

Estadística de fiabilidad por dimensión

\begin{tabular}{l|l|l|} 
Dimensiones & Alfa de Cronbach & Alfa de Cronbach basada en elementos estandarizados \\
Atención & 0,718 & 0,725 \\
Claridad & 0,767 & 0,768 \\
Reparación & 0,781 & 0,778
\end{tabular}

\subsection{Procedimiento de recogida y análisis de datos}

El procedimiento seguido para obtener los datos fue el siguiente: inicialmente, se solicitó la autorización del Jefe de DAEM de Temuco a los directores de las escuelas. Los consentimientos informados se enviaron a las escuelas por oficina de partes, los cuales fueron firmados y devueltos a través del mismo medio. La aplicación 
de la Escala TMMS-24 se realizó a través de una plataforma digital. Los datos se exportaron al formato Excel y posteriormente se procesaron en el programa estadístico Statistical Package for the Social Sciences WIN (IBM, SPSS), versión 22.0.

Tras el estudio psicométrico de confiabilidad del instrumento utilizando el Alfa de Cronbach, se realizó el análisis descriptivo, por medio del cual se obtuvo la distribución porcentual y los estadígrafos de media y desviación estándar por dimensión y género, con un intervalo de confianza del 95\%. Estos fueron presentados en tablas. Posteriormente, para el análisis comparativo de cada dimensión por género, se aplicó la prueba no paramétrica de U de Mann-Whitney y para el análisis relacional, el test estadístico Chi cuadrado de Pearson, ambos a un nivel de significancia alfa $=0,05$.

\section{Resultados}

A continuación, en la Tabla 4, se exponen los resultados obtenidos tras la aplicación del TMMS-24 del modelo IE de Mayer y Salovey para cada dimensión evaluada y su respectivo nivel (bajo, adecuado, alto). Para atención emocional, se observa que el $38,4 \%(n=173)$ de los estudiantes se ubica en el nivel adecuado de IE, para la dimensión claridad emocional, el $55,8 \%(n=251)$ de los encuestados se ubica en el nivel adecuado de IE, finalmente, en cuanto a reparación emocional, el 60,9\% $(n=274)$ se ubicó en el nivel adecuado de IE. Considerando los resultados del IE de forma global, el 51,6\% $(n=232)$ presentó nivel adecuado de IE.

\section{Tabla 4}

Frecuencias y porcentajes para los grados de IE

\begin{tabular}{|c|c|c|c|c|c|c|}
\hline Dimensión & $\begin{array}{l}\text { Grad } \\
\text { inteli } \\
\text { emo } \\
\text { Bajo }\end{array}$ & $\begin{array}{l}\text { de } \\
\text { encia } \\
\text { onal }\end{array}$ & Adec & uado & Alto & \\
\hline & $\mathrm{N}$ & $\%$ & & & $\mathrm{~N}$ & $\%$ \\
\hline Atenc & 128 & 28,4 & 173 & 38,4 & 149 & 33,1 \\
\hline Claridad & 167 & 37,1 & 251 & 55,8 & 32 & 7,1 \\
\hline Reparación & 103 & 22,9 & 274 & 60,9 & 73 & 16,2 \\
\hline IE general & 134 & 29,6 & 232 & 51,6 & 85 & 18,7 \\
\hline
\end{tabular}

La Tabla 5 muestra las puntuaciones promedio y la desviación estándar para cada dimensión y de forma global. En ese sentido, para la dimensión atención emocional, se encontró $25,8 \pm 5,3$; para claridad emocional, $26,5 \pm$ 5,9 ; y para reparación emocional, $28,1 \pm 6,2$. Por su parte, el promedio de la IE global se ubica en $26,8 \pm 5,8$. Dado que estas dimensiones se califican como adecuadas cuando las puntuaciones medias se encuentran entre los valores de 22 y 35 puntos. Por lo tanto, los resultados descriptivos sugieren que la mayor parte de los participantes del estudio se ubican en el nivel adecuado en las tres dimensiones.

Ahora bien, para evaluar si los resultados de la Tabla 5 son estadísticamente significativos al nivel del $5 \%$, se ejecutó la prueba no paramétrica denominada $U$ Mann-Whitney para contrastar el nivel adecuado / no adecuados. Los hallazgos de la U $\left(N_{-}(\right.$Adecuado $\left.)=175 ; N \_(\text {No adecuado })=275\right)=23100,000 ; Z=-0,762$, $p=0,447$ sugieren que no hay diferencias estadísticamente significativas entre los estudiantes que tiene una IE adecuada y los que no tienen una IE adecuada. En consecuencia, no se acepta la primera hipótesis que afirma que "los estudiantes de 5to. básico presentan niveles adecuados de inteligencia emocional en función de las dimensiones de la Escala TMMS-24".

\section{Tabla 5}

Descriptivos según dimensión de IE

\begin{tabular}{|l|l|l|}
\hline Dimensión & Promedio & Desviación estándar \\
Atención & 25,8 & 5,3 \\
Claridad & 26,5 & 5,9 \\
Reparación & 28,1 & 6,2 \\
IE general & 26,8 & 5,8 \\
\hline
\end{tabular}

Seguidamente, se procedió a probar la segunda hipótesis, la cual establece que "las mujeres presentan mejores niveles de IE que los hombres en las tres dimensiones. En función de las puntuaciones totales obtenidas por las mujeres y hombres, se construyó para cada dimensión una variable ordinal que contempla los niveles en cada dimensión. Luego, se ejecutó la prueba U Mann-Whitney para evaluar las diferencias en 
cada nivel de IE respecto al género.

Los resultados de la prueba U Mann-Whitney U (N_Mujeres $=224 ; \mathrm{N} \_$Hombres $\left.=226\right)=13744,500 ; Z=$ $8,920, p=4,66 \mathrm{E}-19$ ) indica que las diferencias entre mujeres y hombres en los niveles de atención emocional son estadísticamente significativas. En efecto, en la Tabla 6 se observa que los hombres presentan mejores niveles de atención en comparación con las mujeres. Por lo tanto, se rechaza la hipótesis que establece que las mujeres tienen mejores niveles de atención emocional que los hombres.

\section{Tabla 6}

Distribución porcentual para los niveles de atención emocional según el género

\begin{tabular}{|l|l|l|l|l|l|l|l|}
\hline & & \multicolumn{2}{l|}{ Género } & \multicolumn{2}{l|}{ Total } \\
& & \multicolumn{2}{l|}{ Femenino } & \multicolumn{2}{l|}{ Masculino } & \multicolumn{2}{l|}{} \\
\multirow{3}{*}{ Atención emocional } & Poca atención & 73 & $32,6 \%$ & 55 & $24,3 \%$ & 128 & $28,4 \%$ \\
& Adecuada atención & 140 & $62,5 \%$ & 33 & $14,6 \%$ & 173 & $38,4 \%$ \\
\multirow{2}{*}{ Total } & Demasiada atención & 11 & $4,9 \%$ & 138 & $61,1 \%$ & 149 & $33,1 \%$ \\
& & 224 & $100 \%$ & 226 & $100 \%$ & 450 & $100 \%$ \\
\hline
\end{tabular}

En cuanto a la dimensión claridad emocional, la U (N_Mujeres $=224 ; \mathrm{N} \_$Hombres $\left.=226\right)=19275,000 ; Z=$ $-4,972, p=6,64 \mathrm{E}-7$ refleja que las diferencias son estadísticamente significativas entre mujeres y hombres. En tal sentido, la Tabla 7 muestra que las mujeres poseen mejores niveles de claridad emocional en comparación con los hombres; por lo tanto, no se rechaza la hipótesis que afirma que las mujeres tienen mejores niveles de claridad emocional que los hombres.

\section{Tabla 7}

Distribución porcentual para los niveles de claridad emocional según el género

\begin{tabular}{|c|c|c|c|c|c|c|c|}
\hline \multirow{5}{*}{ Claridad emocional } & \multirow{5}{*}{$\begin{array}{l}\text { Mejora su claridad } \\
\text { Adecuada claridad } \\
\text { Excelente claridad }\end{array}$} & \multicolumn{4}{|c|}{ Género } & \multicolumn{2}{|c|}{ Total } \\
\hline & & F & $\%$ & & & $\mathrm{~F}$ & $\%$ \\
\hline & & 57 & $25,4 \%$ & 110 & $48,7 \%$ & 167 & $37,1 \%$ \\
\hline & & 147 & $65,6 \%$ & 104 & $46,0 \%$ & 251 & $55,8 \%$ \\
\hline & & 20 & $8,9 \%$ & 12 & $5,3 \%$ & 32 & $7,1 \%$ \\
\hline Total & & 224 & $100,0 \%$ & 226 & $100,0 \%$ & 450 & $100,0 \%$ \\
\hline
\end{tabular}

Para la dimensión regulación emocional, la U $\left(\mathrm{N} \_\right.$Mujeres $=224 ; \mathrm{N} \_$Hombres $\left.=226\right)=23381,000 ; Z=-1,608$, $p=0,108$ muestra que hay diferencias estadísticamente no significativas según el género para los niveles de esta dimensión. Por tanto, a partir de los datos que muestra la Tabla 8, se puede afirmar que las mujeres y los hombres presentan niveles de regulación emocional similares. En consecuencia, se rechaza la hipótesis que las mujeres tienen mejores niveles de regulación emocional que los hombres.

\section{Tabla 8}

Distribución porcentual para los niveles de regulación emocional según el género

\begin{tabular}{|c|c|c|c|c|c|c|c|}
\hline \multirow{5}{*}{$\begin{array}{l}\text { Regulación } \\
\text { emocional }\end{array}$} & \multirow{5}{*}{$\begin{array}{l}\text { Mejorar su regulación } \\
\text { Adecuada regulación } \\
\text { Excelente regulación }\end{array}$} & \multicolumn{2}{|c|}{$\begin{array}{l}\text { Género } \\
\text { Femenino }\end{array}$} & \multicolumn{2}{|c|}{ Masculino } & \multicolumn{2}{|c|}{ Total } \\
\hline & & $\mathrm{F}$ & $\%$ & $\mathrm{~F}$ & & $\mathrm{~F}$ & $\%$ \\
\hline & & 49 & $21,9 \%$ & 54 & $23,9 \%$ & 103 & $22,9 \%$ \\
\hline & & 130 & $58,0 \%$ & 144 & $63,7 \%$ & 274 & $60,9 \%$ \\
\hline & & 45 & $20,1 \%$ & 28 & $12,4 \%$ & 73 & $16,2 \%$ \\
\hline Total & & 224 & $100,0 \%$ & 226 & $100,0 \%$ & 450 & $100,0 \%$ \\
\hline
\end{tabular}

Por otro lado, se comprobó la tercera hipótesis según la cual "No hay relación entre las dimensiones de la IE y el género". Para dar respuesta a esta hipótesis, se usó la prueba $\chi^{2}$ de Pearson con la finalidad de evaluar la relación entre las tres dimensiones de la IE y el género, con un nivel de significancia del $5 \%$. Los resultados de $\chi^{2}(2, N=450)=176,95$ y $p=3,76 E-39$ indican que existen diferencias estadísticamente significativas entre la dimensión atención emocional y el género. Al cuantificar la intensidad de la relación encontrada con el coeficiente de contingencia $(C)$, se obtuvo $C=0,531$ y $p=3,76 \mathrm{E}-39$, lo cual indica un grado medio de relación estadísticamente significativo. A partir del resultado que muestra la Tabla 9, las mujeres tienden a relacionarse con los niveles más bajos de atención emocional y los hombres, con los niveles más altos. Por consiguiente, se rechaza la hipótesis que establece que el género no está relacionado con la dimensión atención emocional. 
Tabla 9

Distribución porcentual para género vs. niveles de atención emocional

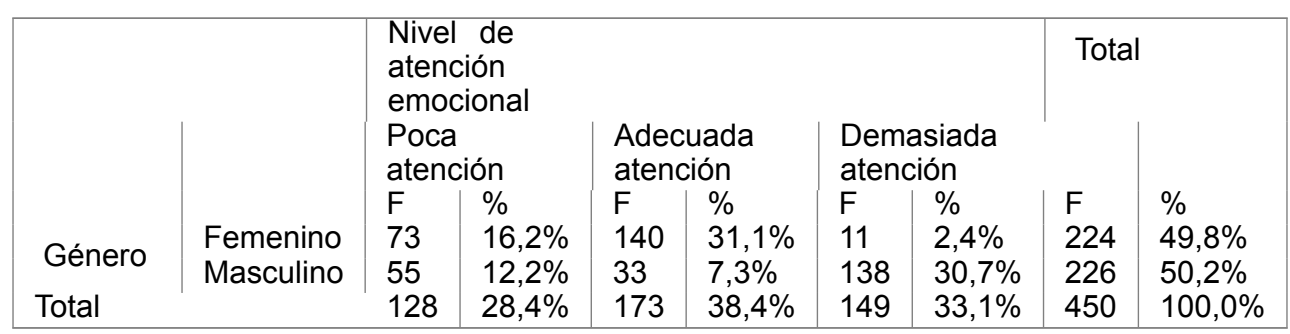

Para la dimensión claridad emocional, la $\chi^{2}(2, N=450)=26,18$ y $\mathrm{p}=0,000002$ indica que existen diferencias estadísticamente significativas entre los niveles de reportados en esta dimensión y el género. Asimismo, al cuantificar la relación, el coeficiente $C=0,234$ y $p=0,000002$ sugiere un grado bajo de relación, pero estadísticamente significativo. Esto se evidencia en la Tabla 10, donde se observa que las mujeres tienen a relacionarse con los niveles más altos de claridad emocional y los hombres, con los niveles más bajos. En este sentido, se rechaza la hipótesis que indica que el género no está relacionado con la dimensión claridad emocional.

Tabla 10

Distribución porcentual para género vs. niveles de claridad emocional

\begin{tabular}{|c|c|c|c|c|c|c|c|c|c|}
\hline & & $\begin{array}{l}\text { Niv } \\
\text { clar } \\
\text { em }\end{array}$ & $\begin{array}{l}\mathrm{de} \\
\text { ad } \\
\text { onal }\end{array}$ & & & & & Tota & \\
\hline & & $\begin{array}{l}\text { Mej } \\
\text { su } \\
\text { clar }\end{array}$ & & $\begin{array}{l}\text { Ade } \\
\text { clari }\end{array}$ & $\begin{array}{l}\text { lada } \\
\text { ad }\end{array}$ & & $\begin{array}{l}\text { lente } \\
\text { dad }\end{array}$ & & \\
\hline & & $\begin{array}{l}F \\
57\end{array}$ & $\%$ & $F$ & $\%$ & $\mathrm{~F}$ & $\%$ & $\mathrm{~F}$ & $\%$ \\
\hline Género & Masculino & $\begin{array}{l}5 / \\
110\end{array}$ & $\begin{array}{l}12,1 \% \\
24.4 \%\end{array}$ & $\begin{array}{l}41 / \\
104\end{array}$ & $\begin{array}{l}23,1 \% \\
23,1 \%\end{array}$ & 12 & $\begin{array}{l}4,4 \% \\
2.7 \%\end{array}$ & 226 & $50,2 \%$ \\
\hline Total & & 167 & $37,1 \%$ & 251 & $55,8 \%$ & 32 & $7,1 \%$ & 450 & $100,0 \%$ \\
\hline
\end{tabular}

Finalmente, respecto a la dimensión regulación emocional, se encontró $\chi^{2}(2, N=450)=4,91$ y $\mathrm{p}=0,086$ que no existen diferencias estadísticamente significativas entre los niveles registrados en esta dimensión y el género. En consecuencia, no fue necesario cuantificar la relación con el coeficiente $C$. Los datos que sustentan esta conclusión se pueden observar en la Tabla 11, donde se muestra que las mujeres y los hombres no muestran una inclinación determinante hacia alguno de los niveles de regulación emocional. Por consiguiente, no se rechaza la hipótesis que indica que el género no está relacionado con la dimensión regulación emocional.

Tabla 11

Distribución porcentual para género vs. niveles de reparación emocional

\begin{tabular}{|c|c|c|c|c|c|c|c|c|c|}
\hline \multirow[b]{2}{*}{$\begin{array}{l}\text { Género } \\
\text { Total }\end{array}$} & \multirow[b]{2}{*}{$\begin{array}{l}\text { Femenino } \\
\text { Masculino }\end{array}$} & \multicolumn{2}{|c|}{$\begin{array}{l}\text { Nivel } \\
\text { reparación } \\
\text { emocional } \\
\text { Mejorar } \\
\text { su } \\
\text { reparación }\end{array}$} & \multicolumn{2}{|c|}{$\begin{array}{l}\text { Adecuada } \\
\text { reparación }\end{array}$} & \multicolumn{2}{|c|}{$\begin{array}{l}\text { Excelente } \\
\text { reparación }\end{array}$} & Tota & \\
\hline & & $\begin{array}{l}F \\
49 \\
54 \\
103\end{array}$ & $\begin{array}{l}\% \\
10,9 \% \\
12,0 \% \\
22,9 \%\end{array}$ & $\begin{array}{l}F \\
130 \\
144 \\
274\end{array}$ & $\begin{array}{l}\% \\
28,9 \% \\
32,0 \% \\
60,9 \%\end{array}$ & $\begin{array}{l}F \\
45 \\
28 \\
73\end{array}$ & $\begin{array}{l}\% \\
10,0 \% \\
6,2 \% \\
16,2 \%\end{array}$ & $\begin{array}{l}F \\
224 \\
226 \\
450\end{array}$ & $\begin{array}{l}\% \\
49,8 \% \\
50,2 \% \\
100,0 \%\end{array}$ \\
\hline
\end{tabular}

\section{Discusión}

Previo a la aplicación del instrumento, se evaluó la consistencia interna del total de preguntas mediante el Alfa de Cronbach; se obtuvo como resultado $87 \%$. Respecto de las medidas obtenidas, estas fueron superiores a $70 \%$, tanto consideradas globalmente como discriminadas por dimensiones. Esto confirma que los 24 ítems 
que conforman la escala son homogéneos y que la escala mide de forma consistente las dimensiones de la IE en estudiantes de 10 a 12 años. Este hallazgo resulta significativo, pues contribuye con el conocimiento de la fiabilidad y validez de esta escala, que se viene usando en inglés desde 1990 y en español desde 1998 (Fernández-Berrocal et al., 1998). En la mayoría de las investigaciones previas, este instrumento se ha empleado con niños mayores de 12 años, jóvenes y adultos; por lo tanto, este estudio aporta para su posterior aplicación en niños de 10 a 12 años. Fernández-Berrocal y Extremera (2005) advierten que la aplicación con niños menores de esas edades, sobre todo menores de 10 años, puede arrojar resultados menos fiables. Sin embargo, considerando que se logró el objetivo planteado, en esta investigación se demostró que el instrumento es aplicable con niños chilenos de 10 a 12 años.

En cuanto a la comprobación de la hipótesis 1: "Los estudiantes de 5to. básico presentan niveles adecuados de inteligencia emocional en función de las dimensiones de la Escala TMMS-24, los hallazgos de este estudio muestran que la mayoría de los estudiantes de 5to. básico se ubican en el nivel adecuado en las tres dimensiones de la Escala TMMS-24 de inteligencia emocional percibida: atención, claridad y regulación. Por otro lado, los hallazgos de la prueba estadística sugieren que no hay diferencias estadísticamente significativas entre los estudiantes que tiene una IE adecuada y los que no tienen una IE adecuada, vistos de manera global. En consecuencia, no se acepta la hipótesis antes señalada.

La tendencia hacia la IE adecuada observada en el grupo de estudiantes coincide con los hallazgos de Katyal y Awasthi (2005), Garay (2014), Rodríguez et al. (2019), Seelvaraj et al. (2020) y Toscano-Hermoso et al. (2020), quienes también encontraron que la mayor parte de los sujetos participantes alcanzó la calificación de adecuado en las dimensiones de la IE.

En tercer lugar, se comprobó la hipótesis 2, que afirma que "Las mujeres presentan mejores niveles de IE que los hombres en las tres dimensiones. Con tal propósito se aplicó la prueba $U$ de Mann Whitney. Los resultados para atención emocional indican que hay diferencias estadísticamente significativas y los hombres alcanzan niveles superiores, por lo cual se rechaza esta hipótesis, lo cual es coincidente con Toscano-Hermoso et al. (2020). Sin embargo, este resultado difiere de los hallazgos de Thayer et al. (2003), Extremera y Fernández (2005), Bastian et al. (2005), Sánchez et al. (2008), Salguero et al. (2010), Gartzia et al. (2012), Garay (2014) y Cabello et al. (2016), quienes hallaron que las mujeres puntúan mejor que los hombres en atención emocional. También, difiere de Gómez et al. (2020), quienes hallaron que las adolescentes chilenas tienen mejores puntuaciones. Por su parte, Cazalla-Luna y Molero (2016) no encontraron diferencias estadísticamente significativas entre géneros en esta dimensión.

En cuanto a la claridad emocional, al comparar la distribución entre hombres y mujeres, se observan diferencias estadísticamente significativas, las mujeres presentan niveles más altos de claridad que los hombres; por consiguiente, se acepta la mencionada hipótesis. Este resultado coincide con los hallazgos de Gartzia et al. (2012) y Cabello et al. (2016) en adultos españoles, pero difiere de Bastian et al. (2005), Salguero et al. (2010), Garay (2014), Cazalla-Luna y Molero (2016), Gómez et al. (2020) y Toscano-Hermoso et al. (2020), quienes no observaron diferencias estadísticamente significativas entre las puntuaciones de claridad emocional en universitarios australianos, adolescentes españoles y chilenos, respectivamente. Este hallazgo se puede explicar considerando la perspectiva generacional actual que sugiere que los roles de hombres y mujeres en la sociedad han cambiado y predomina la flexibilización de roles (Sánchez et al., 2008). Esto permite que cada género comprenda y practique lo que tradicionalmente le correspondía al otro, favoreciendo la validación y valoración entre géneros (Alcalay et al., 2011). Guastello y Guastello (2003) estudiaron los efectos generacionales de padres e hijos sobre los roles de género e inteligencia emocional, hallaron que "los hijos eran más andróginos en personalidad que sus padres" (p. 1).

Por su parte, los resultados del tercer componente relacionado con la hipótesis 2 referido a la reparación emocional indican que no hay diferencias estadísticamente significativas entre hombres y mujeres en la puntuación de esta dimensión; por lo tanto, se rechaza la hipótesis de que las mujeres se ubican en niveles más altos. Los resultados de la atención emocional indican que los hombres obtuvieron niveles más altos que las mujeres. Esto resultados difieren de Salguero et al. (2010), Garay (2014), Cazalla-Luna y Molero (2016) y Gómez et al. (2020), quienes no hallaron diferencias de género estadísticamente significativas en la puntuación de la dimensión reparación emocional en adolescentes españoles, peruanos y chilenos, respectivamente. En cambio, coinciden con Gartzia et al. (2012), Cazalla-Luna y Molero (2016) y Toscano-Hermoso et al. (2020), quienes también encontraron que los hombres obtuvieron mejores resultados que las mujeres en regulación emocional. Cabello et al. (2016), por su parte, observaron diferencias significativas, pero en favor de las mujeres.

Finalmente, se comprobó la hipótesis 3 que plantea que "No hay relación entre las dimensiones de la IE y el género", mediante la aplicación de la prueba $\chi^{2}$ de Pearson para determinar la relación entre las tres dimensiones de la IE y el género. Los resultados indican que existen diferencias estadísticamente significativas entre la dimensión atención emocional y el género, pues las mujeres tienden a ubicarse en los niveles más bajos de atención emocional y los hombres en los más altos. Por consiguiente, se rechaza la hipótesis que 
establece que el género no está relacionado con la dimensión atención emocional. Bindu \& Thomas (2006) hallaron resultados similares en adultos jóvenes. Garay (2014), en cambio, halló resultados opuestos a los del presente estudio. En cuanto a la dimensión claridad emocional, se encontraron diferencias estadísticamente significativas, las mujeres se ubican en los niveles más altos y los hombres, en lo más bajos. Por lo tanto, se rechaza la hipótesis que indica que el género no está relacionado con la dimensión claridad emocional. Los resultados de estas dos dimensiones coinciden con Bindu y Thomas (2006), Ferrándiz et al. (2012), Garay (2014), Cabello et al. (2016) y Rodríguez et al. (2019), quienes encontraron que hay relación estadísticamente significativa entre las variables IE y género. En cambio, en cuanto a la dimensión regulación emocional, no se hallaron diferencias estadísticamente significativas, las mujeres y los hombres no muestran una inclinación determinante hacia alguno de los niveles de regulación emocional; por lo tanto, se acepta la hipótesis que indica que el género no está relacionado con la dimensión regulación emocional. Este resultado coincide con los hallazgos de Goleman (1998), Katyal y Awasthi (2005), Bindu y Thomas (2006), Williams et al. (2009), Garay (2014), McKinley et al. (2014), Cabello et al. (2016), Cazalla-Luna y Molero (2016), Salavera et al. (2017), Fischer et al. (2018) y Mokhlesi y Patil (2018), quienes encontraron que el género no influye significativamente en la IE; pero difiere de Bindu y Thomas (2006), Ferrándiz et al. (2012), Gartzia et al. (2012), Rodríguez et al. (2019) y Seelvaraj et al. (2020), que hallaron una asociación estadísticamente significativa entre ambas variables.

\section{Conclusiones}

La inteligencia emocional es la capacidad de percibir, expresar, comprender y razonar con la emoción, la habilidad de conectar el razonamiento y las emociones cuando se procesa información. Estudios previos han indicado que esta inteligencia está asociada al género (Salovey \& Mayer, 1990; Mayer \& Salovey, 1993; Goleman, 1995, 1998). Por ello, en procura de analizar el comportamiento de estas variables en el contexto educativo chileno, esta investigación buscó comparar las dimensiones de IE atención, claridad y regulación emocional de la Escala TMMS-24 de Mayer y Salovey y el género en un grupo de estudiantes de 5to. básico de las escuelas públicas de Temuco.

En primer lugar, se encontró que los estudiantes de 5to. básico de educación primaria tienden, de forma global, a ubicarse en niveles adecuados de IE.

En segundo lugar, se intentó probar que las mujeres se ubicaban en niveles más altos que los hombres. Los resultados permiten aceptar esta hipótesis solo para dimensión claridad emocional; las dimensiones atención y reparación emocional, en cambio, indican que las mujeres no se ubican en niveles superiores a los hombres. En tercer lugar, se buscó comprobar la hipótesis que establece que no existe relación entre los niveles obtenidos por los estudiantes en cada dimensión y el género. En este sentido, se rechaza esa hipótesis para las dimensiones atención y claridad emocional, pues se hallaron diferencias estadísticamente significativas; en cambio, para la dimensión reparación emocional, se comprueba que no existe relación entre los niveles obtenidos por los estudiantes en cada dimensión y el género, pues hombres y mujeres se comportaron de manera similar.

En definitiva, no hay un claro predomino en las valoraciones entre hombres y mujeres en las tres dimensiones analizadas, pues no se halló una clara tendencia que indique que las mujeres se ubican en niveles más altos que los hombres en las tres dimensiones. Tampoco hay datos concluyentes para establecer categóricamente que las valoraciones de los estudiantes en las tres dimensiones están relacionadas con el género. Estos resultados pueden estar relacionados con que la selección de la muestra no se realizó empleando procedimientos estadísticos para evitar el sesgo. El hecho de que sea una muestra intencional pudo haber afectado los resultados. Por lo tanto, es necesario hacer más investigaciones con escolares chilenos de entre 10 y 12 años, en las cuales tanto el tamaño de la muestra como los procedimientos para su selección y distribución se realicen mediante técnicas estadísticas.

Por otro lado, este estudio analiza cuantitativamente la inteligencia emocional, lo cual puede limitar su comprensión. Por lo tanto, como lo afirma Rom Harré (Belli et al., 2020), es necesario estudiar cualitativamente las emociones desde la perspectiva de la psicología social, considerando variables socioculturales de los estudiantes, pues las emociones están condicionadas por un componente psicológico, uno cognitivo y uno social. Los resultados de estos estudios podrían explicar el fenómeno de las emociones y la inteligencia emocional de forma integral (Palmer et al., 2005; Pal \& Baruah, 2021).

También, se podrían desarrollar proyectos de investigación para analizar la relación entre IE y género considerando las características de las escuelas participantes.

Como propuesta de acción, se podría diseñar un plan de educación emocional al DAEM, para fortalecer la inteligencia emocional en sus tres dimensiones en grupos de estudiantes más grandes. Siguiendo a Güell (2014), esto permitiría desarrollar mayores niveles de creatividad, motivación, conocimiento de sí mismo y, en 
definitiva, mejorar la convivencia. La inclusión de estas competencias emocionales junto con las estrategias de enseñanza aprendizaje activas y participativas deberían dar como resultado mejores niveles de aprendizaje en los estudiantes.

Finalmente, considerando la propuesta de Marchant et al. (2015) sobre la importancia de capacitar en competencias emocionales a directivos y docentes de estudiantes de 3ro. a $7 \mathrm{mo}$. básico en Santiago de Chile, sería conveniente incluir la IE en los programas de formación docente inicial y educación continua, que les permita a los docentes y al personal directivo abordar sistemáticamente el área socioemocional en el curriculum e influir positivamente en la educación de los estudiantes. Para estos autores, la capacitación es el "camino estratégico para potenciar y promover el desarrollo socioemocional de los niños y jóvenes" (p. 216). Pensando en las proyecciones del presente artículo y considerando que la educación en el siglo XXI conlleva responsabilidades compartidas entre la escuela y la familia, se hace necesario abordar la enseñanza de la inteligencia emocional formalmente en la escuela. En este sentido, Fernández-Berrocal y Extremera (2002) plantean que las organizaciones y grupos desarrollan un clima emocional propio determinado en un alto porcentaje por la IE de los líderes de las escuelas. Por lo tanto, con los resultados de esta investigación, se puede proponer una investigación sobre IE y liderazgo de los equipos directivos de las escuelas, o bien, las estrategias de enseñanza aprendizaje vinculadas con la IE implementadas por los docentes de las escuelas.

\section{Apoyos}

Universidad de Sevilla

\section{Referencias}

Alcalay, L., Milicic, N., Torretti, A., y Berger, C. (2011). ¿Coeducación o educación segregada por sexo? Una aproximación desde la perspectiva de la educación de género. Revista Psykhe, 9(2), 171-179. https://doi.org/ 10.7764/psykhe.v9i2.20197

Ángela Díaz-Herrero, Gonzálvez, C., Sanmartín, R., Vicent, M., Martín, N. L.-S., Inglés, C. J., y GarcíaFernández, J. M. (2018). Profiles of emotional intelligence and demotivation to attend school in Chilean adolescents. Motivation and Emotion, 42(6), 947-959. https://doi.org/10.1007/s11031-018-9712-4

Bastian, V. A., Burns, N. R., y Nettelbeck, T. (2005). Emotional intelligence predicts life skills, but not as well as personality and cognitive abilities. Personality and Individual Differences, 39(6), 1135-1145. https://doi.org/ $10.1016 /$ j. paid.2005.04.006

Belli, S., Aceros, J. C., y Harré, R. (2020). ¡Todo es discursivo!” cruzando fronteras y dialogando con Rom Harré. Psicologia \& Sociedade, 32, 1-16. https://doi.org/10.1590/1807-0310/2020v32193420

Billings, C. E., Downey, L. A., Lomas, J. E., Lloyd, J., y Stough, C. (2014). Emotional Intelligence and scholastic achievement in pre-adolescent children. Personality and Individual Differences, 65, 14-18. https://doi.org/ 10.1016/j.paid.2014.01.017

Bindu, P., y Thomas, I. (2006). Gender differences in emotional intelligence. Psychological Studies-University of Calicut, 51(4), 261-268. Retrieved from https://t.ly/XwU2

Brody, L. R., y Hall, J. (2000). A Gender, emotion, and expression. In M. Lewis (Ed.), Handbook of emotions (p. 338-349). Guilford.

Cabello, R., Sorrel, M. A., Fernández-Pinto, I., Extremera, N., y Fernández-Berrocal, P. (2016). Age and gender differences in ability emotional intelligence in adults: A cross-sectional study. Developmental Psychology, 52(9), 1486-1492. https://doi.org/10.1037/dev0000191

Cazalla-Luna, N., y Molero, D. (2015). Inteligencia emocional percibida, disposición al optimismo-pesimismo, satisfacción vital y personalidad de docentes en su formación inicial. Revista de Investigación Educativa, 34(1), 241-241. https://doi.org/10.6018/rie.34.1.220701

de-la Educación, A.-C. (2017). Retrieved from http://www.agenciaorienta.cl/autoridades-basica de-la Salud, O.-M. (2021). Temas de salud. Género. Retrieved from https://t.ly /hbHz

Extremera, N., y Berrocal, P. (2005). Inteligencia emocional percibida y diferencias individuales en el metaconocimiento de los estados emocionales: una revisión de los estudios con el TMMS. Ansiedad y estrés, 11(2-3), 101-122. Retrieved from https://t.ly/GMqq

Extremera, N., Fernández-Berrocal, P., y Salovey, P. (2006). Spanish version of the Mayer-Salovey-Caruso Emotional Intelligence Test (MSCEIT). Psicothema, 18, 42-48. Retrieved from https://www.redalyc.org/pdf/ 727/72709506.pdf

Fernández-Berrocal, P., Alcaide, R., Domínguez, E., Fernández-Mcnally, C., Ramos, N. S., y Rovira, M. (1998). Adaptación al castellano de la escala rango de metaconocimiento sobre estados emocionales de Salovey et al.: datos preliminares. In Libro de Actas del V Congreso de Evaluación Psicológica.

Fernández-Berrocal, P., Cabello, R., Castillo, R., y Extremera, N. (2012). Gender differences in emotional 
intelligence: The mediating effect of age. Behavioral Psychology, 20(1), 77-89. Retrieved from https://t.ly/ CNGO

Fernández-Berrocal, P., y Extremera, N. (2005). La inteligencia emocional y la educación de las emociones desde el modelo de Mayer y Salovey. Revista Interuniversitaria de Formación del Profesorado, 19(3), 63-93. Retrieved from https:// bit.ly/2B67y0x

Fernandez-Berrocal, P., Extremera, N., y Ramos, N. (2004). Validity and Reliability of the Spanish Modified Version of the Trait Meta-Mood Scale. Psychological Reports, 94(3), 751-755. https://doi.org/10.2466/pr0.94.3 $.751-755$

Fernández-Berrocal, P., y Extremera-Pacheco, N. (2002). La inteligencia emocional como una habilidad esencial en la escuela. Revista Iberoamericana de Educación, 29(1), 1-6. https://doi.org/10.35362/rie2912869 Ferrándiz, C., Hernández, D., Berjemo, R., Ferrando, M., y Sáinz, M. (2012). Social and emotional intelligence in childhood and adolescence: Spanish validation of a measurement instrument // La inteligencia emocional y social en la niñez y adolescencia: validación de un instrumento para su medida en lengua castellana. Revista de Psicodidactica / Journal of Psychodidactics, 17(2), 309-338. Retrieved from https://dx.doi.org/10.1387/ revpsicodidact.2814 10.1387 /revpsicodidact.2814

Ferres, M. A., de Luna, E. B., y Sánchez, M. J. (2017). Estudio sobre la inteligencia emocional y los factores contextuales en estudiantes de cuarto de educación primaria de la provincia de Granada. Revista de Investigación Educativa, 36(1), 141-141. https://doi.org/10.6018/rie.36.1.281441

Fischer, A. H., Kret, M. E., y Broekens, J. (2018). Gender differences in emotion perception and self-reported emotional intelligence: A test of the emotion sensitivity hypothesis. PLOS ONE, 13(1), e0190712-e0190712. https://doi.org/10.1371/journal.pone.0190712

Fragoso-Luzuriaga, R. (2015). Inteligencia emocional y competencias emocionales en educación superior ¿un mismo concepto? Revista Iberoamericana de Educación Superior, 6(16). https://doi.org/10.22201/iisue .20072872e.2015.16.154

Garay, C. (2014). Inteligencia emocional y su relación con el rendimiento académico de los estudiantes del sexto ciclo de la Facultad de Educación de la Universidad Nacional de San Marcos. Universidad Nacional de San Marcos. Retrieved from https://t.ly/Y17x

García-Fernández, J. M., Inglés, C. J., Suriá, R., Martín, N. L.-S., Gonzálvez-Maciá, C., Aparisi, D., y MartínezMonteagudo, M. C. (2015). Profiles of emotional intelligence and learning strategies in a sample of Chilean students. European Journal of Psychology of Education, 30(4), 437-455. https://doi.org/10.1007/s10212-015 $-0254-9$

Gartzia, L., Aritzeta-Galan, A., Balluerka-Lasa, N., y Barbera-Heredia, E. (2012). Inteligencia emocional y género: más allá de las diferencias sexuales. Anales de Psicología, 28(2), 567-575. https://doi.org/10.6018/ analesps.28.2.124111

Goleman, D. (1995). Emotional intelligence. Bantam Books.

Goleman, D. (1997). Emotional intelligence: Why it can matter more than IQ. Bantom Books.

Goleman, D. (1998). Working with emotional intelligence. Bantam Books.

Gómez-Núñez, M. I., Torregrosa, M. S., Inglés, C. J., Martín, N. G. L. S., Sanmartín, R., Vicent, M., y GarcíaFernández, J. M. (2020). Factor Invariance of the Trait Meta-Mood Scale-24 in a Sample of Chilean Adolescents. Journal of Personality Assessment, 102(2), 231-237. https://doi.org/10.1080/00223891.2018.1505730

Guastello, D. D., y Guastello, S. J. (2003). Androgyny, Gender Role Behavior, and Emotional Intelligence Among College Students and Their Parents. Sex Roles, 49(11/12), 663-673. https://doi.org/10.1023/b:sers.0000003136 .67714 .04

Güell, M. (2014). ¿ ¿Tengo inteligencia emocional? Paidós.

Hernández, R., Fernández, C., y Baptista, P. (2014). Metodología de la investigación (6ª́Edición ed.). McGraw Hill.

Herrera-Torres, L., Buitrago-Bonilla, R. E., y Cepero-Espinosa, S. (2017). Emotional Intelligence in Colombian Primary School Children. Location and Gender Analysis. Universitas Psychologica, 16(3), 1-1. https://doi.org/ 10.11144/javeriana.upsy16-3.eips

Jausovec, N., y Jausovec, K. (2005). Sex differences in brain activity related to general and emotional intelligence. Brain and Cognition, 59, 277-286. https://doi.org/10.1016/j.bandc.2005.08.001

Joseph, D. L., y Newman, D. A. (2010). Emotional intelligence: An integrative meta-analysis and cascading model. Journal of Applied Psychology, 95(1), 54-78. https://doi.org/10.1037/a0017286

Katyal, S., y Awasthi, E. (2005). Gender Differences in Emotional Intelligence Among Adolescents of Chandigarh. Journal of Human Ecology, 17(2), 153-155. https://doi.org/10.1080/09709274.2005.11905771

Khan, M. A., y Bhat, N. A. (2013). Emotional intelligence of adolescent boys and girls. International Journal of Educational Research and Development, 2(3), 67-71. Retrieved from https://t.ly/o3Ca

Lillo, J. (2004). Crecimiento y comportamiento en la adolescencia. Revista de la Asociación Española de Neuropsiquiatría, 90, 57-71. https://doi.org/10.4321/s0211-57352004000200005

Marchant, T., Milicic, N., y Alamos, P. (2015). Competencias socioemocionales, capacitación de directivos y 
docentes y su impacto en la autoestima de alumnos de $3^{\circ}$ a $7^{\circ}$ básico. Revista Iberoamericana de Evaluación Educativa, 8(2), 203-218. Retrieved from https://bit.ly/2UH7Txy

Martínez-Marín, M., y Martínez, C. (2016). Relación entre la inteligencia emocional y género. In J. Soler, L. Moreno, O. Díaz, E. Escolano, y A. Rodríguez (Eds.), Inteligencia emocional y bienestar II, Reflexiones, experiencias profesionales e investigaciones (p. 622-636). Ediciones Universidad San Jorge. Retrieved from https://bit.ly/3fo5oYY

Maturana, H. (2001). Emociones y lenguaje en educación y política. Ediciones Dolmen.

Mayer, J. D., y Salovey, P. (1993). The intelligence of emotional intelligence. Intelligence, 17(4), 433-442. https://doi.org/10.1016/0160-2896(93)90010-3

McKinley, S. K., Petrusa, E. R., Dijk, C. F.-V., Mullen, J. T., Smink, D. S., Scott-Vernaglia, S. E., Kent, T. S., Black-Schaffer, W. S., y Phitayakorn, R. (2014). Are There Gender Differences in the Emotional Intelligence of Resident Physicians? Journal of Surgical Education, 71(6), e33-e40. https://doi.org/10.1016/j.jsurg.2014.05 .003

Merchán, I. (2017). Test de Habilidad de Inteligencia Emocional en la Escuela (THInEmE). Universidad de Extremadura. Retrieved from https://bit.ly/37sfuVJ

Mokhlesi, V., y Patil, C. B. (2018). A study of gender differences in emotional Intelligence and learning behaviour among children. The International Journal of Indian Psychology, 6(4), 2349-3429. Retrieved from https://t.ly/Vlev

Pal, S., y Baruah, M. (2021). An assessment of the role of gender on emotional intelligence and creative problem-solving ability for healthy adolescents. Zeichen Journal, $7(1)$. Retrieved from https://t.ly/6huT

Palmer, B. R., Gignac, G., Manocha, R., y Stough, C. (2005). A psychometric evaluation of the MayerSalovey-Caruso Emotional Intelligence Test Version 2.0. Intelligence, 33, 285-305. https://doi.org/10.1016/ j.intell.2004.11.003

Pulido, F., y Herrera, F. (2017). Habilidades emocionales a lo largo del desarrollo educativo. Revista Científica Electrónica de Educación y Comunicación en la Sociedad del Conocimiento, 1(17), 91-106. Retrieved from https://t.ly/RBTC

Rodríguez, J., Sánchez, R., Ochoa, L., Cruz, I., y Fonseca, R. (2019). Niveles de inteligencia emocional según género de estudiantes en la educación superior. Revista Espacios(31), 40-40. Retrieved from https://bit.ly/ 3ebr7my

Rothman, A. J., y Salovey, P. (1997). Shaping perceptions to motivate healthy behavior: The role of message framing. In P. Salovey y D. Sluyter (Eds.), Psychological Bulletin (Vol. 121, p. 3-19). American Psychological Association (APA). https://doi.org/10.1037/0033-2909.121.1.3

Ruiz, R. (2013). Inteligencia emocional: bases históricas, fundamentos biológicos y posibles aplicaciones. Retrieved from https://t.ly/sS4J

Salavera, C., Usán, P., y Jarie, L. (2017). Emotional intelligence and social skills on self-efficacy in Secondary Education students. Are there gender differences? Journal of Adolescence, 60, 39-46. https://doi.org/10.1016/ j.adolescence.2017.07.009

Salguero, J. M., Extremera, N., y Fernández-Berrocal, P. (2012). Emotional intelligence and depression: The moderator role of gender. Personality and Individual Differences, 53(1), 29-32. https://doi.org/10.1016/ j.paid.2012.02.006

Salguero, J. M., Fernández-Berrocal, P., Balluerka, N., y Aritzeta, A. (2010). Measuring Perceived Emotional Intelligence in the Adolescent Population: Psychometric Properties of the Trait Meta-Mood Scale. Social Behavior and Personality: an international journal, 38(9), 1197-1209. https://doi.org/10.2224/sbp.2010.38.9 .1197

Salovey, P., y Mayer, J. D. (1990). Emotional Intelligence. Imagination, Cognition and Personality, 9(3), 185-211. https://doi.org/10.2190/dugg-p24e-52wk-6cdg

Salovey, P., Mayer, J. D., Goldman, S. L., Turvey, C., y Palfai, T. P. (1995). Emotional attention, clarity, and repair: Exploring emotional intelligence using the Trait Meta-Mood Scale (J. W. Pennebaker, Ed.). American Psychological Association. https://doi.org/10.1037/10182-006

Sánchez, M., Fernández-Berrocal, Montañez, J., y Latorre, J. (2008). ¿Es la inteligencia emocional una cuestión de género? Socialización de las competencias emocionales en hombre y mujeres y sus implicaciones. Education \& Psychology, 6(15), 455-474. https://doi.org/10.25115/ejrep.v6i15.1287

Santesso, L. D., Dana, L. R., Schmidt, L. A., y Segalowitz, S. J. (2006). Frontal Electroencephalogram Activation Asymmetry, Emotional Intelligence, and Externalizing Behaviors in 10-Year-Old Children. Child Psychiatry and Human Development, 36(3), 311-328. https://doi.org/10.1007/s10578-005-0005-2

Seelvaraj, A. M., Panda, B. K., y Sinha, M. (2020). Emotional intelligence in children of Christian missionary schools at Kolkata. MIER Journal of Educational Studies Trends \& Practices, 10(2), 267-277. Retrieved from https://t.ly/ivun

Shahzad, S., y Bagum, N. (2012). Gender differences in trait emotional intelligence: A comparative study. Business Review, 7(2), 106-112. Retrieved from https://t.ly/sDKW 
Thayer, J. F., Rossy, L. A., Padial, E. R., y Johnsen, B. H. (2003). Gender differences in the relationship between emotional regulation and depressive symptoms. Cognitive Therapy and Research, 27, 349-364. https://doi.org/10.1023/A:1023922618287

Toscano-Hermoso, M. D., Ruiz-Frutos, C., Fagundo-Rivera, J., Gómez-Salgado, J., García-Iglesias, J. J., y Romero-Martín, M. (2020). Emotional Intelligence and Its Relationship with Emotional Well-Being and Academic Performance: The Vision of High School Students. Children, 7(12), 310-310. https://doi.org/10.3390/ children 7120310

Traveset, M. (2016). Pensar con el corazón, sentir con la mente. Octaedro.

Ugarriza, N., y del Águila, L. P. (2005). La evaluación de la inteligencia emocional a través del inventario de BarOn ICE: NA, en una muestra de niños y adolescentes. Revista Persona, O(008), 11-11. https://doi.org/ 10.26439/persona2005.n008.893

Valadez, M., Borges, M., Ruvalcaba, N., Villegas, K., y Lorenzo, M. (2013). La Inteligencia emocional y su relación con el género, el rendimiento académico y la capacidad intelectual del alumnado universitario. Electronic Journal of Research in Educational Psychology, 11(30), 395-412. https://doi.org/10.14204/ejrep.30 .12204

Williams, C., Daley, D., Burnside, E., y Hammond-Rowley, S. (2009). Measuring Emotional Intelligence in preadolescence. Personality and Individual Differences, 47(4), 316-320. https://doi.org/10.1016/j.paid.2009.03 .019 